\title{
The effects of essential oil mouthrinses with or without alcohol on plaque and gingivitis: a randomized controlled clinical study
}

Michael C. Lynch', Sheila Cavalca Cortelli², James A. McGuire', Jane Zhang ${ }^{1 *}$ (D, Danette Ricci-Nittel ${ }^{1}$, Carolyn J. Mordas ${ }^{1}$, Davi Romeiro Aquino ${ }^{2}$ and Jose Roberto Cortelli ${ }^{2}$

\begin{abstract}
Background: The efficacy of several variants of essential oil mouthrinses has been studied extensively. This is the first study to compare the anti-plaque and anti-gingivitis efficacy of two marketed essential oil mouthrinses: one is an alcohol containing mouthrinse and the other one is an alcohol-free mouthrinse.

Methods: This examiner-blind, parallel-group study randomized subjects to three groups: 1) Mechanical Oral Hygiene $(\mathrm{MOH})$ only; 2) $\mathrm{MOH}$ plus Alcohol-Containing essential oil Mouthrinse (ACM); 3) $\mathrm{MOH}$ plus Alcohol-Free essential oil Mouthrinse (AFM). Primary endpoint was whole-mouth mean Modified Gingival Index (MGI) at six months. Secondary endpoints included whole-mouth mean MGI at one and three months, and whole-mouth mean Plaque Index (PI) and whole-mouth mean Bleeding Index (BI) at one, three and six months. Safety assessments were conducted at all time points.

Results: A total of 370 subjects were enrolled; 348 subjects completed the study. After six months, subjects using essential oil mouthrinses with or without alcohol showed significant reduction $(p<0.001)$ in gingivitis $(28.2 \%$ and 26. $7 \%$, respectively) and significant reduction $(p<0.001)$ in plaque $(37.8 \%$ and $37.0 \%$, respectively), compared to those performing $\mathrm{MOH}$ only. Significant reductions in $\mathrm{MGI}, \mathrm{PI}$, and $\mathrm{BI}(p<0.001)$ were observed at one and three months and also at six months for mean BI. No statistically significant differences were observed for all measured indices between ACM and AFM groups at any time point. Both mouthrinses were well tolerated.
\end{abstract}

Conclusions: No significant differences were observed in the efficacy of ACM and AFM to reduce plaque and gingivitis, when used in addition to $\mathrm{MOH}$, over six months.

Trial registration: The trial was registered on clinicaltrials.gov on November 30, 2016. The registration number is NCT02980497.

Keywords: Essential oils, Mouthrinse, Alcohol-free, Plaque, Gingival bleeding, Gingivitis

\footnotetext{
*Correspondence: jzhang1@its.jnj.com

'Johnson \& Johnson Consumer Inc.., 199 Grandview Road, Skillman, New

Jersey 08558, USA

Full list of author information is available at the end of the article
} 


\section{Background}

When used in conjunction with mechanical oral hygiene $(\mathrm{MOH})$, the use of mouthrinses to help reduce plaque and gingivitis has been well documented and their efficacy is attributed to chemical agents, such as Cetylpyridinium Chloride (CPC), Chlorhexidine (CHX), and Essential Oils (EO) contained in these products [1-9]. Commonly used inactive ingredients include alcohol, water, buffering agents, flavouring agents and surfactants. In EO mouthrinses, alcohol serves as a preservative but also solubilizes the essential oils, thus maintaining their bioavailability $[10,11]$. A 2015 meta-analysis of 29 clinical trials with durations of six months or longer further demonstrated the efficacy of essential oil mouthrinses [7].

The inclusion of alcohol in mouthrinse has historically limited its use in certain patient populations, such as children, alcoholics, people with strong taste preferences, those of certain religious beliefs, and patients with oral mucositis [12].

To address these limitations, an Alcohol-Free EOmouthrinse (AFM) was developed. Its antimicrobial properties were demonstrated in vitro [13] and in vivo [14] initially. This was followed by demonstration of its efficacy in reducing plaque and gingivitis in clinical trials $[15,16]$. However, no long-term studies (i.e., at least six months in duration) have directly compared alcohol containing EO mouthrinses (ACM) to its Alcohol-Free counterpart, AFM. The objective of the current study is to compare the long-term efficacy of twice-daily rinsing with either an AFM or an $\mathrm{ACM}$, in conjunction with usual $\mathrm{MOH}$, for the reduction of plaque and gingivitis.

\section{Methods}

\section{Study design}

This randomized, controlled, single-centre, examinerblind, parallel-group study was conducted between 11 September 2013 and 16 March 2014 (recruitment period July 2013-September 2013) at the University of Taubate, São Paulo, Brazil. The study was conducted in accordance with the International Conference on Harmonisation (ICH) Harmonised Tripartite Guideline for Good Clinical Practice (ICH E6, 1996), in agreement with the Declaration of Helsinki (2000), applicable local regulations, and the American Dental Association (ADA) Seal of Acceptance Program Guidelines for Chemotherapeutic Products for Control of Gingivitis [17]. The study protocol was approved by the Institutional Ethics Committee on research involving humans (CAAE: 20,402,713.1.00005501). The trial was registered on clinicaltrials.gov. The registration number is NCT02980497. Written informed consent was obtained from all subjects.

\section{Subjects}

Subjects from Taubate and neighbouring areas were selected from a database or recruited through advertising and were consecutively enrolled in the study. Males and females aged $\geq 18$ years, in good general and oral health, with the exception of mild to moderate gingivitis, and with a minimum of 20 natural teeth having scorable facial and lingual surfaces, were included. Each subject was required to have a whole-mouth mean gingivitis score of $\geq 1.95$, according to the Modified Gingival Index (MGI) [18], and a whole-mouth mean plaque score of $\geq 1.95$, according to the Turesky modification of the Quigley-Hein Plaque index (PI) [19] on six surfaces per tooth [20]. Other study inclusion criteria included the absence of moderate/advanced periodontitis (ADA types III and IV), absence of significant oral soft tissue pathology other than plaque-induced gingivitis; and no fixed or removable orthodontic appliance or removable dentures. Periodontitis patients were excluded based on Tanner's early periodontitis definition [21], which required one or more periodontal sites with $>2 \mathrm{~mm}$ periodontal attachment loss in a given subject with mean clinical attachment level $\leq 1.5 \mathrm{~mm}$. Key exclusion criteria included regular use of products such as triclosan dentifrices and mouthrinses containing $\mathrm{EO}, \mathrm{CPC}$, or $\mathrm{CHX}$ within two weeks prior to baseline; use of antibiotic, anti-inflammatory, or anticoagulant therapy during the study or within two weeks prior to baseline; history of significant adverse effects, including allergies following use of oral hygiene products; known sensitivity to the investigational product ingredients; current, or history of alcohol or drug abuse; and participation in a clinical trial within 30 days of the start of the study.

\section{Interventions}

At baseline, after abstaining from oral hygiene for at least eight hours, but no more than $18 \mathrm{~h}$, pre-screened subjects underwent an oral examination, and gingivitis and plaque assessments. A disclosing dye $(0.5 \%$ basic fuchsin neutral red solution [Byoformula, Taubate, São Paulo, Brazil]) was used to assess plaque. Eligible subjects received a dental prophylaxis to remove plaque, calculus and stain, and were randomly assigned to one of the three treatment groups:

1) a negative control group, or a Mechanical Oral Hygiene $(\mathrm{MOH})$ only group, received a fluoridated toothpaste (Colgate Cavity Protection, Colgate-Palmolive Company, New York, USA) and $\mathrm{REACH}^{\circ}$ Soft-bristled toothbrush (Johnson \& Johnson, New Jersey USA);

2) a test group received the same toothpaste and toothbrush, plus LISTERINE ${ }^{\circ}$ ZERO $^{\mathrm{im}}$, an Alcohol Free Mouthrinse (AFM) (Johnson \& Johnson, New Jersey, USA);

3) a positive control group received the same toothpaste and toothbrush, plus LISTERINE ${ }^{\circ}$ COOL MINT', 
an Alcohol Containing Mouthrinse (ACM) (Johnson \& Johnson, New Jersey, USA).

Both AFM and ACM contain a fixed combination of four essential oils [eucalyptol (0.092\%), menthol (0.042\%), methyl salicylate $(0.060 \%)$, and thymol $(0.064 \%)]$, as well as sorbitol, poloxamer, buffer, flavour and dye. AFM also contains Sodium Lauryl Sulphate and propylene glycol.

At the start of the study, all subjects received $\mathrm{REACH}^{\circ}$ Soft-bristled toothbrush, Colgate ${ }^{\circ}$ anticavity fluoride toothpaste and were instructed to brush with one ribbon of toothpaste in their usual manner twice daily. Colgate anticavity fluoride toothpaste contained Sodium Monofluorophosphate $(0.76 \%$ or $0.15 \% w / v$ Fluoride Ion), Dicalcium Phosphate Dihydrate, Water, Glycerin, Sodium Lauryl Sulfate, Cellulose Gum, Flavor, Tetrasodium Pyrophosphate, Sodium Saccharin. Subjects in the ACM or AFM groups also received the assigned mouthrinse and plastic dosage cups marked at $20 \mathrm{~mL}$ level and were instructed to rinse with $20 \mathrm{~mL}$ of full strength mouthrinse for $30 \mathrm{~s}$ after brushing twice daily. All subjects received diaries to document compliance with the homecare regimen. Diaries were checked monthly and bottles were weighed monthly to match the number of rinses reported in the diaries to the actual used volume. Absence of $>3$ consecutive rinses or $>5$ rinses within each 30-day interval between study visits constituted a protocol violation and the subject could be removed from the study at the discretion of the clinical investigator or the Sponsor.

Randomization was based on a block randomization scheme devised by the Biometrics and Clinical Data Systems Department at Johnson \& Johnson, with a block size of six. Each subject was assigned a unique randomization number that determined the treatment assigned to that subject according to a randomization schedule. The randomization number was assigned sequentially in ascending order and could not be reassigned to another subject. Investigational supplies were kept separate from the site personnel involved in examining the subjects. The dental examiner was, therefore, blinded to treatment throughout the study period. Subjects received investigational products in blinded packaging, and personnel dispensing the investigational supplies or supervising their use did not participate in the examination of subjects to minimize potential bias. The first rinsing and the rinsing on the examination visits were under supervision at the study site. All other rinsing was unsupervised.

\section{Assessments and outcomes}

Efficacy assessments were performed at baseline and at the one-, three- and six-month visits. This involved visual assessment of gingival inflammation, supragingival plaque, and gingival bleeding, as measured by MGI, PI, and gingival bleeding index (BI) [22, 23], respectively. Subjects were asked to abstain from oral hygiene for at least eight hours, but no more than $18 \mathrm{~h}$ prior to their clinical examinations.

A trained and calibrated dental examiner performed all examinations in the following order: oral tissues assessment, MGI, BI and PI. One examiner was used throughout the study.

Gingivitis was assessed using the MGI on the buccal and lingual marginal gingiva and interdental papillae of all scorable teeth as follows: 0 - normal (absence of inflammation); 1 - mild inflammation of any portion of the gingival unit; 2 - mild inflammation of the entire gingival unit; 3 - moderate inflammation of the gingival unit; 4 -severe inflammation of the gingival unit.

To assess gingival bleeding, a periodontal probe was inserted into the gingival crevice, and swept from distal to mesial around the tooth at an angle of approximately $60^{\circ}$, while in contact with the sulcular epithelium. Each of four gingival areas (distobuccal, mid-buccal, midlingual, and mesiolingual) around each tooth was assessed. After approximately $30 \mathrm{~s}$, bleeding at each gingival unit was recorded according to the following scale: 0 - absence of bleeding after $30 \mathrm{~s} ; 1$ - bleeding after 30 s; 2 - immediate bleeding.

The plaque area was scored on six surfaces per tooth [20] (distobuccal, midbuccal and mesiobuccal, distolingual, midlingual and mesiolingual) of all scorable teeth as follows: 0 - no plaque; 1 - separate flecks or discontinuous band of plaque at the gingival margin; 2 - up to $1 \mathrm{~mm}$ continuous band of plaque at the gingival margin; 3 - band of plaque wider than $1 \mathrm{~mm}$ but less than $1 / 3$ of surface; 4 - plaque covering $1 / 3$ or more, but less than $2 / 3$ of surface; 5 - plaque covering $2 / 3$ or more of surface.

Safety assessments included oral examinations conducted at baseline, and at monthly visits, to monitor the effect of the mouthrinse formulations on soft and hard tissues. Changes from the baseline and previous visits were recorded at each subsequent clinic visit. Clinically significant findings were recorded as adverse events (AEs) and an assessment was made regarding relationship to investigational product at the discretion of a medically qualified clinical examiner.

During the study, subjects were instructed to follow their usual dietary habits and normal oral care regimen, incorporating only the toothpaste, toothbrush, and mouthrinse provided to them. Subjects were also allowed to continue use of an interdental cleaning device to remove impacted food between the teeth if it was part of their usual oral care regimen. No other oral hygiene procedures were permitted, including teeth cleaning or dental work, except in an emergency.

The primary efficacy endpoint was the mean MGI at six months, while the secondary endpoints included 


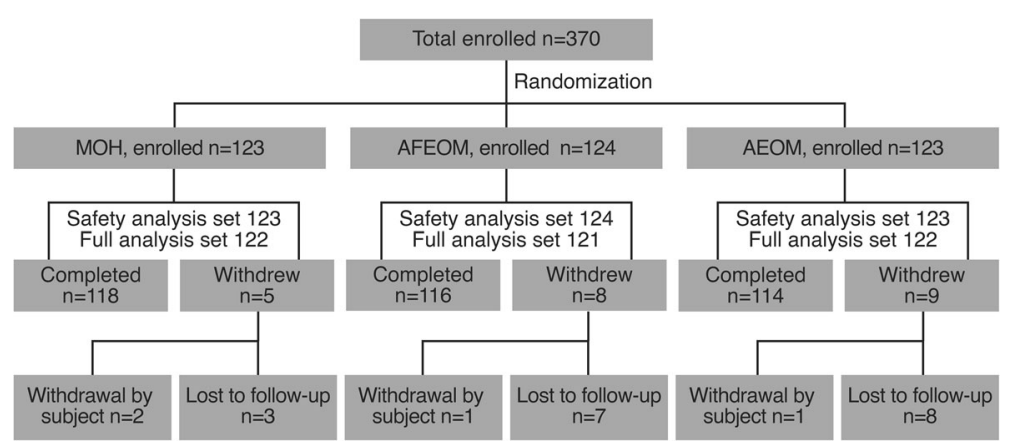

Fig. 1 Subject disposition/CONSORT flow diagram. MOH: mechanical oral hygiene; AFM: alcohol-free essential oil mouthrinse; ACM: alcohol-containing essential oil mouthrinse

mean MGI at one and three months; and mean PI and $\mathrm{BI}$ at one, three, and six months. Safety was assessed by summarizing all AEs considered related to treatment.

\section{Statistical analyses}

The planned sample size of 330 subjects (110 per treatment group) provided $90 \%$ power to detect a betweentreatment difference of 0.08 , assuming a standard deviation [SD] of 0.180 , based on previous studies $[16,24]$ for whole-mouth mean MGI at a 0.05 significance level (two-sided). Demographic and baseline characteristics were compared across treatment groups using analysis of variance (ANOVA), Chi-square test, or Fisher's exact test. Efficacy analysis was based on the full analysis set (FAS), following the Intent-to-Treat principle, defined as all randomized subjects who used the study product and had baseline and at least one post-baseline data point for mean MGI. Missing data was assumed Missing At Random. No imputation of missing data was performed. Statistical comparisons for primary and secondary variables were based on one-way analysis of covariance (ANCOVA), with treatment as a factor and the

Table 1 Subject demographic and baseline characteristics

\begin{tabular}{|c|c|c|c|c|c|}
\hline Parameters & $\begin{array}{l}\mathrm{MOH} \\
(n=123)\end{array}$ & $\begin{array}{l}\text { AFM } \\
(n=124)\end{array}$ & $\begin{array}{l}\text { ACM } \\
(n=123)\end{array}$ & $\begin{array}{l}\text { Total } \\
(n=370)\end{array}$ & $\begin{array}{l}\text { Overall } \\
p \text {-value }\end{array}$ \\
\hline Age (years) & $38.8 \pm 11.75$ & $35.1 \pm 14.43$ & $35.2 \pm 13.83$ & $36.4 \pm 13.46$ & $0.053^{a}$ \\
\hline Sex n (\%) & & & & & $0.757^{b}$ \\
\hline Male & $50(40.7)$ & $46(37.1)$ & $51(41.5)$ & $147(39.7)$ & \\
\hline Female & $73(59.3)$ & $78(62.9)$ & $72(58.5)$ & $223(60.3)$ & \\
\hline Race n (\%) & & & & & $>0.999^{c}$ \\
\hline White & $112(91.1)$ & $113(91.1)$ & $112(91.1)$ & $337(91.1)$ & \\
\hline Black or African- American & $1(<1.0)$ & $1(<1.0)$ & $1(<1.0)$ & $3(<1.0)$ & \\
\hline Asian & $1(<1.0)$ & 0 & 0 & $1(<1.0)$ & \\
\hline Other & $9(7.3)$ & $10(8.1)$ & $10(8.1)$ & $29(7.8)$ & \\
\hline Smoker n (\%) & & & & & $0.876^{b}$ \\
\hline Yes & $13(10.6)$ & $11(8.9)$ & $11(8.9)$ & $35(9.5)$ & \\
\hline No & $110(89.4)$ & $113(91.1)$ & $112(91.1)$ & $335(90.5)$ & \\
\hline \multicolumn{6}{|l|}{ Whole-mouth scores } \\
\hline Baseline Mean MGl & $2.439 \pm 0.147$ & $2.454 \pm 0.149$ & $2.462 \pm 0.146$ & $2.452 \pm 0.147$ & $0.450^{\mathrm{a}}$ \\
\hline Baseline Mean PI & $2.976 \pm 0.224$ & $2.991 \pm 0.228$ & $3.014 \pm 0.269$ & $2.994 \pm 0.241$ & $0.456^{a}$ \\
\hline Baseline Mean Bl & $0.604 \pm 0.132$ & $0.628 \pm 0.183$ & $0.623 \pm 0.163$ & $0.618 \pm 0.161$ & $0.474^{\mathrm{a}}$ \\
\hline
\end{tabular}

Data are mean \pm SD unless otherwise specified

ACM Alcohol-containing essential oil mouthrinse, AFM Alcohol-free essential oil mouthrinse, $B$ l Bleeding index, $M G I$ Modified gingival index, $M O H$ Mechanical oral hygiene, PI Plaque index, SD Standard deviation

${ }^{a} p$-values are based on ANOVA model with term for treatment

${ }^{\mathrm{b}} p$-values are based on Chi-square test

'based on Fisher's exact test 
corresponding baseline value as a covariate. Comparisons were made in the following sequential manner to control the family-wise error rate of 0.05: ACM versus $\mathrm{MOH}$ with respect to mean MGI; AFM versus $\mathrm{MOH}$ with respect to mean MGI and; ACM versus AFM with respect to mean MGI. All comparisons were made using a 0.05 level test (two-sided) and secondary endpoints were analysed in the same manner. Safety analysis was based on all randomized subjects who used the study product.

\section{Post-hoc analysis}

An exploratory post-hoc analysis was conducted to evaluate the non-inferiority (defined as "at least as good as") of AFM compared with ACM. Using Fieller's theorem [25, 26], 90\% confidence intervals (CIs) for the ratio of observed mean MGI scores of AFM and ACM at months 1, 3, and 6 were constructed. According to ADA guidelines (American Dental Association Council on Scientific Affairs 2011), the criterion for non-inferiority was satisfied when the entire 90\% Fieller CI consisted of values no greater than $110 \%$.

\section{Results}

Of the 370 subjects enrolled in the study and randomized, 348 completed the study, while 22 withdrew or were lost to follow up (Fig. 1). There were no protocol violations leading to removal from the study. In total, 123, 124, and 123 subjects were included in the $\mathrm{MOH}$, AFM, and ACM groups, respectively; 122, 121, and 122 subjects who had both baseline and post-baseline data were included in the FAS. The mean (SD) age of subjects was 36.4 (13.46) years and the majority were females (60.3\%), Caucasian (91.1\%), and non-smokers (90.5\%). Overall, mean (SD) MGI, PI, and BI were 2.452 (0.147), $2.994(0.241)$, and 0.618 (0.161), respectively. Baseline and demographic characteristics were comparable between the three study groups (Table 1).

\section{Efficacy}

Primary endpoint

At six months, ACM use resulted in a $28.2 \%$ reduction (between treatment difference $[95 \% \mathrm{CI}] ; p$ value) $(-0.62$ $[-0.670,-0.570] ; p<0.001)$ in gingivitis, as measured by MGI, compared to $\mathrm{MOH}$. AFM showed a $26.7 \%$ reduction $(-0.59[-0.637,-0.538] ; p<0.001)$ in mean MGI

Table 2 Whole-mouth mean modified gingival index (MGI) at months 1, 3, and 6 (full analysis set)

\begin{tabular}{|c|c|c|c|}
\hline Mean MGl & $\begin{array}{l}\mathrm{MOH} \\
(n=122)\end{array}$ & $\begin{array}{l}\text { AFM } \\
(n=121)\end{array}$ & $\begin{array}{l}\text { ACM } \\
(n=122)\end{array}$ \\
\hline \multicolumn{4}{|l|}{ Baseline } \\
\hline Mean \pm SE & $2.438 \pm 0.013$ & $2.452 \pm 0.013$ & $2.463 \pm 0.013$ \\
\hline \multicolumn{4}{|l|}{ Month 1} \\
\hline Adj. mean $\pm S E$ & $2.191 \pm 0.014$ & $1.832 \pm 0.014$ & $1.838 \pm 0.014$ \\
\hline \multicolumn{2}{|c|}{ Difference between treatments (95\% Cl) } & Percent difference (\%) & $p$-value ${ }^{a}$ \\
\hline AFM vs $\mathrm{MOH}$ & $-0.359(-0.399,-0.319)$ & -16.4 & $<0.001$ \\
\hline ACM vs $\mathrm{MOH}$ & $-0.353(-0.393,-0.313)$ & -16.1 & $<0.001$ \\
\hline ACM vs AFM & $0.006(-0.034,0.046)$ & 0.3 & 0.756 \\
\hline \multicolumn{4}{|l|}{ Month $3^{b}$} \\
\hline Adj. mean \pm SE & $2.257 \pm 0.018$ & $1.772 \pm 0.018$ & $1.768 \pm 0.018$ \\
\hline \multicolumn{2}{|c|}{ Difference between treatments (95\% Cl) } & Percent difference (\%) & $p$-value \\
\hline AFM vs $\mathrm{MOH}$ & $-0.486(-0.535,-0.437)$ & -21.5 & $<0.001$ \\
\hline ACM vs $\mathrm{MOH}$ & $-0.489(-0.539,-0.440)$ & -21.7 & $<0.001$ \\
\hline ACM vs AFM & $-0.004(-0.053,0.045)$ & -0.2 & 0.878 \\
\hline \multicolumn{4}{|l|}{ Month $6^{c}$} \\
\hline Adj. mean \pm SE & $2.201 \pm 0.018$ & $1.614 \pm 0.018$ & $1.581 \pm 0.018$ \\
\hline \multicolumn{2}{|c|}{ Difference between treatments (95\% Cl) } & Percent difference (\%) & $p$-value \\
\hline AFM vs $\mathrm{MOH}$ & $-0.587(-0.637,-0.538)$ & -26.7 & $<0.001$ \\
\hline ACM vs $\mathrm{MOH}$ & $-0.620(-0.670,-0.570)$ & -28.2 & $<0.001$ \\
\hline ACM vs AFM & $-0.033(-0.083,0.017)$ & -2.0 & 0.198 \\
\hline
\end{tabular}

ACM Alcohol-containing essential oil mouthrinse, AFM Alcohol-free essential oil mouthrinse, Cl Confidence interval, MGI Modified gingival index, MO Mechanical oral hygiene, SE Standard error

${ }^{a} p$-values are based on ANCOVA model with term for treatment and baseline value as covariate

${ }^{b}$ At month 3, data for $\mathrm{MOH}, \mathrm{AFM}$, and ACM groups were obtained from 119 subjects in each group

${ }^{c}$ At month 6, data for $\mathrm{MOH}, \mathrm{AFM}$, and ACM groups were obtained from 118, 116, and 114 subjects, respectively 
versus $\mathrm{MOH}$. However, no significant difference in gingivitis reduction was found between ACM and AFM treatments after six months of use $(-0.033[-0.083$, 0.017]; $p=0.198$ ) (Table 2).

\section{Secondary endpoints}

Statistically significant reductions in gingivitis compared to $\mathrm{MOH}$ were also seen at one and three months in both the ACM and AFM mouthrinse groups (Table 2). Similar to the results at six months, no significant differences were observed in gingivitis reduction between the two mouthrinse groups at one month $(0.006$ $[-0.034,0.046] ; p=0.756)$ or three months $(-0.004$ $[-0.053,0.045] p=0.878)$ of use.

Both mouthrinses significantly reduced plaque and gingival bleeding compared to $\mathrm{MOH}$ at months one, three, and six $(p<0.001)$ (Tables 3 and 4). No significant difference was seen in the reduction in plaque or gingival bleeding between ACM and AFM groups at one, three, and six months. Figure 2 illustrates the reduction in whole-mouth mean MGI, PI, and BI over the study duration across all treatment groups.

\section{Post hoc analysis}

A post-hoc statistical analysis was conducted to demonstrate that AFM is "at least as good as" ACM. The ratio of least square means of whole-mouth MGI between AFM and ACM at six months was 1.021 or $102 \%(90 \%$ Fieller CI [99\%, 105\%]) (Table 5).

\section{Safety}

All treatments were well tolerated by the subjects. The reported AEs were mild or moderate in severity and no serious AEs were reported, nor did any subject withdraw from the study due to AEs. Three subjects (0.8\%) experienced AEs that were judged as related to the study product. This included one subject each in the ACM and AFM groups who experienced sensitivity of teeth, and one subject in the ACM group who reported dyspepsia. All investigation product-related AEs resolved without any treatment.

\section{Discussion}

Historically, a key concern with formulating EO mouthrinses without alcohol was the subsequent reduction in the solubility of these essential oils, leading to their

Table 3 Whole-mouth mean plaque index (PI) at months 1, 3, and 6 (full analysis set)

\begin{tabular}{|c|c|c|c|}
\hline Mean PI & $\mathrm{MOH}(n=122)$ & $\operatorname{AFM}(n=121)$ & $\operatorname{ACM}(n=122)$ \\
\hline \multicolumn{4}{|l|}{ Baseline } \\
\hline Mean \pm SE & $2.976 \pm 0.020$ & $2.991 \pm 0.021$ & $3.017 \pm 0.024$ \\
\hline \multicolumn{4}{|l|}{ Month 1} \\
\hline Adj. mean \pm SE & $2.781 \pm 0.024$ & $2.186 \pm 0.024$ & $2.206 \pm 0.024$ \\
\hline \multicolumn{2}{|c|}{ Difference between treatments (95\% Cl) } & Percent difference (\%) & $p$-value ${ }^{a}$ \\
\hline AFM vs $\mathrm{MOH}$ & $-0.595(-0.662,-0.527)$ & -21.4 & $<0.001$ \\
\hline $\mathrm{ACM}$ vs $\mathrm{MOH}$ & $-0.574(-0.641,-0.507)$ & -20.6 & $<0.001$ \\
\hline ACM vs AFM & $0.021(-0.047,0.088)$ & 1.0 & 0.544 \\
\hline \multicolumn{4}{|l|}{ Month $3^{b}$} \\
\hline Adj. mean \pm SE & $2.806 \pm 0.022$ & $2.084 \pm 0.022$ & $2.032 \pm 0.022$ \\
\hline \multicolumn{2}{|c|}{ Difference between treatments (95\% Cl) } & Percent difference (\%) & $p$-value ${ }^{a}$ \\
\hline AFM vs $\mathrm{MOH}$ & $-0.723(-0.785,-0.661)$ & -25.8 & $<0.001$ \\
\hline ACM vs $\mathrm{MOH}$ & $-0.775(-0.837,-0.712)$ & -27.6 & $<0.001$ \\
\hline ACM vs AFM & $-0.052(-0.114,0.010)$ & -2.5 & 0.102 \\
\hline \multicolumn{4}{|l|}{ Month $6^{c}$} \\
\hline Adj. mean $\pm S E$ & $2.881 \pm 0.019$ & $1.815 \pm 0.019$ & $1.791 \pm 0.019$ \\
\hline \multicolumn{2}{|c|}{ Difference between treatments (95\% Cl) } & Percent difference (\%) & $p$-value ${ }^{a}$ \\
\hline AFM vs $\mathrm{MOH}$ & $-1.065(-1.118,-1.012)$ & -37.0 & $<0.001$ \\
\hline $\mathrm{ACM}$ vs $\mathrm{MOH}$ & $-1.090(-1.143,-1.036)$ & -37.8 & $<0.001$ \\
\hline ACM vs AFM & $-0.024(-0.078,0.029)$ & -1.3 & 0.371 \\
\hline
\end{tabular}

ACM Alcohol-containing essential oil mouthrinse, AFM Alcohol-free essential oil mouthrinse, $\mathrm{Cl}$ Confidence interval, $M G I$ Modified gingival index, $M O H$ Mechanical oral hygiene, SE Standard error

a $p$-values are based on ANCOVA model with term for treatment and baseline value as covariate

${ }^{\mathrm{b}}$ At month 3, data for $\mathrm{MOH}, \mathrm{AFM}$, and ACM groups were obtained from 119 subjects in each group

${ }^{c}$ At month 6, data for $\mathrm{MOH}, \mathrm{AFM}$, and ACM groups were obtained from 118, 116, and 114 subjects, respectively 
Table 4 Whole-mouth mean gingival bleeding index (BI) at months 1, 3, and 6 (full analysis set)

\begin{tabular}{|c|c|c|c|}
\hline Mean BI & $\mathrm{MOH}(n=122)$ & $\operatorname{AFM}(n=121)$ & $\operatorname{ACM}(n=122)$ \\
\hline \multicolumn{4}{|l|}{ Baseline } \\
\hline Mean \pm SE & $0.605 \pm 0.012$ & $0.630 \pm 0.017$ & $0.623 \pm 0.015$ \\
\hline \multicolumn{4}{|l|}{ Month 1} \\
\hline Adj. mean $\pm S E$ & $0.447 \pm 0.008$ & $0.309 \pm 0.008$ & $0.304 \pm 0.008$ \\
\hline \multicolumn{2}{|c|}{ Difference between treatments ( $95 \% \mathrm{Cl}$ ) } & \multicolumn{2}{|l|}{$p$-value ${ }^{a}$} \\
\hline AFM vs $\mathrm{MOH}$ & $-0.137(-0.160,-0.114)$ & \multicolumn{2}{|l|}{$<0.001$} \\
\hline ACM vs $\mathrm{MOH}$ & $-0.142(-0.165,-0.119)$ & \multicolumn{2}{|l|}{$<0.001$} \\
\hline ACM vs AFM & $-0.005(-0.028,0.018)$ & \multicolumn{2}{|l|}{0.657} \\
\hline \multicolumn{4}{|l|}{ Month $3^{b}$} \\
\hline Adj. mean $\pm S E$ & $0.545 \pm 0.009$ & $0.299 \pm 0.009$ & $0.304 \pm 0.009$ \\
\hline \multicolumn{2}{|c|}{ Difference between treatments $(95 \% \mathrm{Cl}$ ) } & \multicolumn{2}{|l|}{$p$-value ${ }^{a}$} \\
\hline AFM vs $\mathrm{MOH}$ & $-0.246(-0.271,-0.221)$ & $<0.001$ & \\
\hline ACM vs $\mathrm{MOH}$ & $-0.241(-0.266,-0.216)$ & $<0.001$ & \\
\hline ACM vs AFM & $0.006(-0.019,0.030)$ & 0.661 & \\
\hline \multicolumn{4}{|l|}{ Month $6^{c}$} \\
\hline Adj. mean \pm SE & $0.477 \pm 0.007$ & $0.180 \pm 0.007$ & $0.185 \pm 0.007$ \\
\hline \multicolumn{2}{|c|}{ Difference between treatments (95\% Cl) } & \multirow{4}{*}{\multicolumn{2}{|c|}{$p$-value }} \\
\hline AFM vs $\mathrm{MOH}$ & $-0.297(-0.316,-0.278)$ & & \\
\hline ACM vs $\mathrm{MOH}$ & $-0.292(-0.311,-0.272)$ & & \\
\hline ACM vs AFM & $0.005(-0.014,0.025)$ & & \\
\hline
\end{tabular}

ACM Alcohol-containing essential oil mouthrinse, AFM Alcohol-free essential oil mouthrinse, $\mathrm{Cl}$ Confidence interval, $M G I$ Modified gingival index, $\mathrm{MOH}$ Mechanical oral hygiene, SE Standard error

${ }^{a} p$-values are based on ANCOVA model with term for treatment and baseline value as covariate

${ }^{\mathrm{b}}$ At month 3, data for MOH, AFM, and ACM groups were obtained from 119 subjects in each group

'At month 6, data for MOH, AFM, and ACM groups were obtained from 118,

116 , and 114 subjects, respectively

diminished bioactivity and ultimately antimicrobial efficacy. The formulation of an alcohol-free EO mouthrinse therefore required the replacement of alcohol with equally effective solubilisers, while maintaining a balance between all active and inactive ingredients for optimal antimicrobial efficacy.

Contrary to common perception, rinsing with a vehicle that contains alcohol at levels found in mouthrinses, does not provide any clinical benefit. For example, Lamster, et al. [27] demonstrated that a vehicle rinse containing the same amount of alcohol (26.9\%) as a marketed ACM performed similarly to rinsing with a water control after six months of use. In that supervised clinical trial, the ACM group had significantly greater reductions in plaque and gingivitis levels when compared to the alcohol vehicle and water control groups. These findings have since been further validated in a systematic review and meta-analysis [28].

To develop the AFM, systematic evaluation of multiple non-alcoholic delivery systems in saliva-derived mixed-

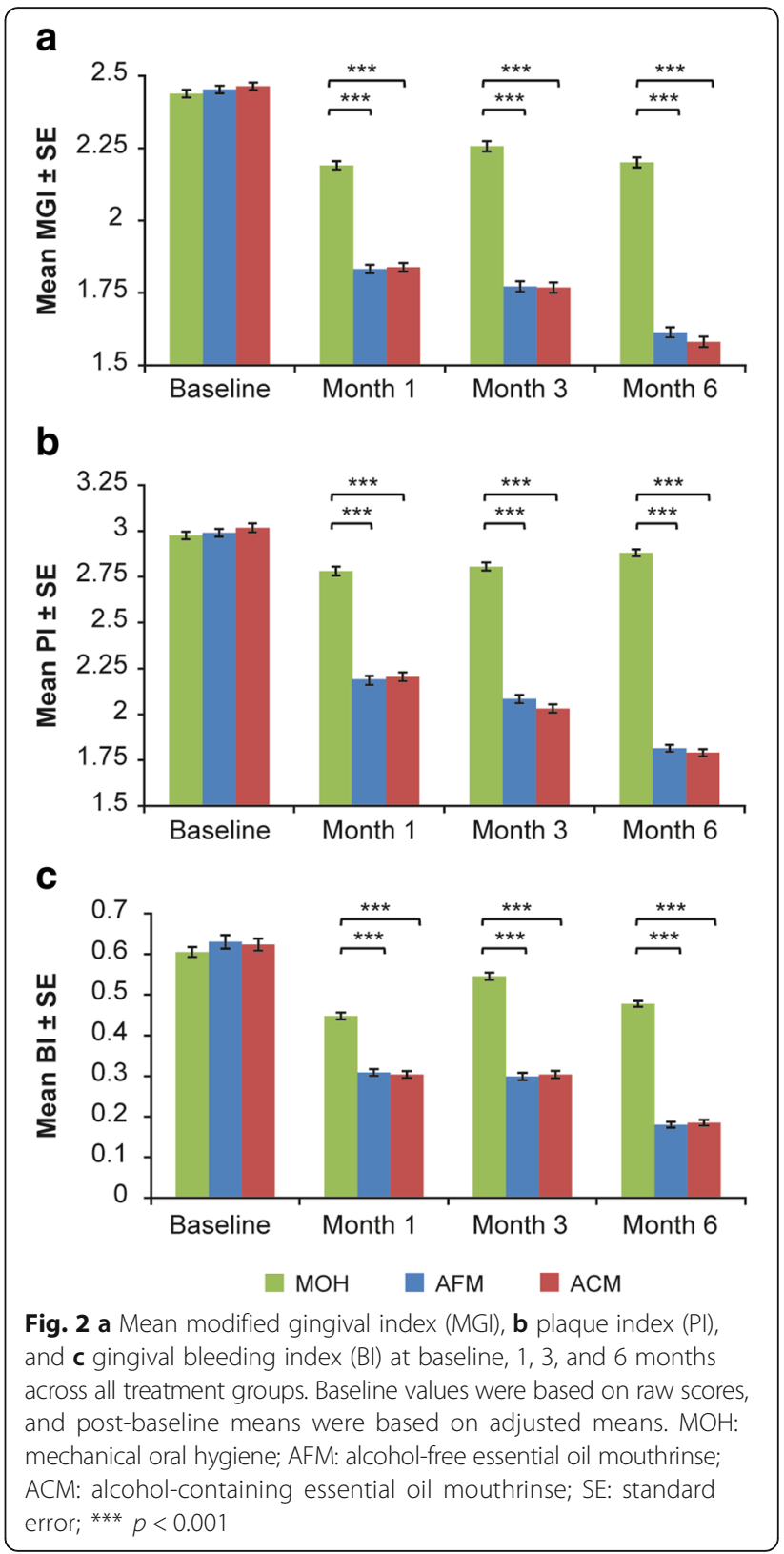

species in vitro biofilm models [29] helped identify the precise combination of a sodium lauryl sulphate, poloxamer, propylene glycol, and sorbitol delivery system that was effective in maintaining bioavailability and bioactivity of EOs. At a range of $0.20 \%-0.35 \% \mathrm{w} / \mathrm{w}$ sodium lauryl sulphate, and a total oil phase content of approximately $0.36 \% \mathrm{w} / \mathrm{w}$, the propylene glycol concentration was found to be optimum between $5 \%$ and $13 \% \mathrm{w} / \mathrm{w}$, while sorbitol was optimum between $10 \%$ and $25 \% \mathrm{w} / \mathrm{w}$ (when added as a 70\% sorbitol solution) [30, 31] Both AFM and ACM contain a fixed combination of four essential oils [eucalyptol $(0.092 \%)$, menthol $(0.042 \%)$, methyl salicylate $(0.060 \%)$, and thymol $(0.064 \%)]$, as well as other formulation ingredients. 
Table 5 Post-hoc analysis: Fieller confidence interval (Cl) for whole-mouth mean gingival index

\begin{tabular}{|c|c|c|c|}
\hline \multirow[t]{2}{*}{ Treatment } & \multicolumn{3}{|l|}{ Mean MGI } \\
\hline & Month 1 & Month 3 & Month 6 \\
\hline AFM & $1.832 \pm 0.0144$ & $1.772 \pm 0.0176$ & $1.614 \pm 0.0179$ \\
\hline ACM & $1.838 \pm 0.0144$ & $1.768 \pm 0.0177$ & $1.581 \pm 0.0181$ \\
\hline AFM/ACM (90\% Cl) & $0.997(0.978,1.015)$ & $1.002(0.979,1.026)$ & $1.021(0.994,1.048)$ \\
\hline
\end{tabular}

Data are adj. Mean \pm standard error. ACM Alcohol-containing essential oil mouthrinse, AFM Alcohol-free essential oil mouthrinse, CI Confidence interval;

${ }^{a}$ Results are based on ANCOVA model with term for treatment and baseline value as covariate

The first proof-of-principle, human study on AFMs used a two-week, no-oral-hygiene, experimental gingivitis model in which the AFM group showed a reduction in plaque by $23.9 \%$ and gingivitis by $10.4 \%$, compared to the negative control rinse group [15]. However, while the results provided an indication of product performance, the restrictions on mechanical oral hygiene limit the applicability of the results.

It is typical to demonstrate the efficacy of a mouthrinse in long-term clinical trials of at least six months in duration, a time period consistent with common recommendations to patients for regular dental visits. A recent meta-analysis identified 35 clinical trials of sixmonth or longer on EO mouthrinses [7] and included 29 trials that met the inclusion criteria. Two of these 29 studies included an AFM but it was not compared with ACM. One of these two studies was published [16]. In this published six-month study, the efficacy of AFM was assessed in comparison to a negative control mouthrinse and an alcohol-free $0.05 \%$ CPC mouthrinse. Subjects performed their daily mechanical oral hygiene $(\mathrm{MOH})$. The EO mouthrinse significantly reduced plaque (31.6\%) and gingivitis $(24.0 \%)$ at six months, compared to the control rinse, and was found to be superior to the CPC mouthrinse. The results of the current study show that both the AFM and ACM provide a statistically and clinically significant additional benefit in subjects who performed their daily $\mathrm{MOH}$. After one month, use of either the AFM or ACM showed significant reduction in plaque $(21.4 \%$ and $20.6 \%$, respectively) and gingivitis (16.4\% and $16.1 \%$, respectively) compared to $\mathrm{MOH}$ alone. By month six, further reduction in plaque was noted; up to $37.0 \%$ and $37.8 \%$ in the AFM and ACM groups, respectively, and gingivitis reduction up to $26.7 \%$ and $28.2 \%$, respectively.

No significant difference was found between the ACM and AFM in plaque and gingivitis reduction $(p=0.371$ and $p=0.198$, respectively) or in gingival bleeding ( $p=$ 0.590), reinforcing the evidence that this alcohol-free delivery system was successful in retaining the EO activity to prevent and reduce gingivitis and plaque.

While the post-hoc analysis should be judged with the caveat that it was exploratory in nature, the results $(90 \%$ Fieller CI $[99 \%, 105 \%]$ ) suggest that since the upper $90 \%$
Fieller CI is below $110 \%$ as specified by the ADA Guidelines for Determination of Efficacy in Product Evaluation (American Dental Association Council on Scientific Affairs 2011), AFM is at least as good as ACM, adding to the evidence supporting the use of AFM. Thus, the inclusion of either ACM or AFM to an individual's oral hygiene regimen can play a valuable role in reducing plaque, gingivitis, and gingival bleeding beyond using $\mathrm{MOH}$ alone. Overall, improving patients' oral hygiene may also help to improve gingival inflammation.

A limitation of this study is the absence of a placebo rinse in the $\mathrm{MOH}$ control group. This was due to the inability to provide a placebo rinse within the timeframe the clinical site had available to conduct the study. The bias introduced by the placebo effect in clinical trials has been well documented [32,33], so it is possible that the act of rinsing/not rinsing itself may have had some modest effect on outcomes. However, the results of the $\mathrm{MOH}$ group in the current study are similar to results from control groups using mechanical oral hygiene plus placebo mouthrinses in previously published studies [16, 34-37] and other studies have shown that placebo rinses with alcohol were not statistically different from water control rinses.

\section{Conclusions}

Alcohol-free and alcohol-containing EO mouthrinses were able to reduce plaque, gingivitis, and gingival bleeding in comparison to the use of mechanical oral hygiene alone in a six-month, randomized study. No significant differences in efficacy in reducing plaque, gingivitis and gingival bleeding were found between alcohol containing and alcohol free essential oil mouthrinse formulations.

\section{Abbreviations}

ACM: Alcohol-containing mouthrinse; ADA: American Dental Association; AE: Adverse Event; AFM: Alcohol-free mouthrinse; ANCOVA: Analysis of covariance; ANOVA: Analysis of Variance; BI: Bleeding index; CHX: Chlorhexidine; Cl: Confidence Interval; CPC: Cetylpyridinium Chloride; EO: Essential Oils; FAS: Full Analysis Set; MGI: Modified Gingival Index; MOH: Mechanical oral hygiene; PI: Plaque index; SD: Standard Deviation

\section{Acknowledgments}

Editorial support, in the form of medical writing, assembling tables based on authors' detailed directions, collating author comments, copyediting, fact checking, and referencing, was provided by Michelle Rebello, PhD, and Namita Bose, PhD, of Cactus Communications, and was funded by Johnson and Johnson Consumer Inc. The authors would like to acknowledge Lisa Fitzgerald for managing the study, Jose Pelino for supporting the study, 
Ratna Revankar for supporting study design and statistical analysis, and Mary Lynn Bosma for critically reviewing the manuscript.

\section{Funding}

Funding for this study was provided by Johnson \& Johnson Consumer Inc.

\section{Availability of data and materials}

The datasets used and/or analysed during the current study available from the corresponding author on reasonable request.

\section{Authors' contributions}

Dr. Lynch (MCL), Dr. Sheila Cortelli (SCC), Ms. Ricci-Nittel (DRN), Dr. Aquino (DRA) and Dr. Jose Cortelli (JRC) contributed to the design and execution of the study. Mr. McGuire (JAM) contributed to the study design and statistical analysis. Dr. Mordas contributed (CJM) to the formulation and related discussion. Dr. Zhang (JZ) led the development of the manuscript and served as the corresponding author. All authors contributed to the development of the manuscript and approved the manuscript.

\section{Ethics approval and consent to participate}

The study protocol was approved by the Institutional Ethics Committee on research involving humans (CAAE: 20,402,713.1.00005501), University of Taubate, Taubaté, São Paolo, Brazil. Written informed consent was obtained from all subjects in agreement with the Declaration of Helsinki.

\section{Consent for publication}

Not applicable.

\section{Competing interests}

Michael Lynch, Danette Ricci-Nittel, James McGuire, Carolyn J. Mordas, and Jane Zhang are employees of Johnson \& Johnson Consumer Inc. Sheila Cavalca Cortelli, Davi Romeiro Aquino and Jose Roberto Cortelli are employees of University of Taubate, Taubaté, São Paolo, Brazil and have received grants from Johnson \& Johnson Consumer Inc. to conduct this study. The authors declare that they have no competing interests.

\section{Publisher's Note}

Springer Nature remains neutral with regard to jurisdictional claims in published maps and institutional affiliations.

\section{Author details}

${ }^{1} J o h n s o n$ \& Johnson Consumer Inc 199 Grandview Road, Skillman, New Jersey 08558, USA. ${ }^{2}$ Nucleus of Periodontal Research, Dental School, University of Taubate, Taubaté, São Paolo, Brazil.

Received: 17 May 2017 Accepted: 10 December 2017

Published online: 10 January 2018

\section{References}

1. Escribano M, Fiquero E, Martín C, Tobías A, Serrano J, Roldán S, Herrera D. Efficacy of adjunctive anti-plaque chemical agents: a systematic review and network meta-analyses of the Turesky modification of the Quigley and Hein plaque index. Journal of Clinical Periodontology. 2016;43:1059-73.

2. Gunsolley JC. A meta-analysis of six-month studies of antiplaque and antigingivitis agents. The journal of the American dental association. 2006; 137:1649-57.

3. Gunsolley JC. Clinical efficacy of antimicrobial mouthrinses. Journal of dentistry 2010;Vol. 38, pp. Suppl 1, S6-10.

4. Haps S, Slot D, Berchier C, van der Weijden G. The effect of cetylpyridinium chloride-containing mouth rinses as adjuncts to toothbrushing on plaque and parameters of gingival inflammation: a systematic review. International Journal of Dental Hygiene. 2008;6:290-303.

5. Serrano J, Escribano M, Roldán S, Martín C, Herrera D. Efficacy of adjunctive anti-plaque chemical agents in managing gingivitis: a systematic review and meta-analysis. Journal of clinical periodontology. 2015:42:S106-38.

6. Van Leeuwen M, Slot D, van der Weijden G. Essential oils compared to chlorhexidine with respect to plaque and parameters of gingival inflammation: a systematic review. Journal of periodontology. 2011;82: 174-94

7. Araujo MW, Charles CA, Weinstein RB, McGuire JA, Parikh-Das AM, Du Q Zhang J, Berlin JA, Gunsolley JC. Meta-analysis of the effect of an essential oil-containing mouthrinse on gingivitis and plaque. The journal of the American dental association. 2015;146:610-22.

8. Chapple ILC, Van der Weijden F, Doerfer C, Herrera D, Shapira L, Polak D. Primary prevention of periodontitis: managing gingivitis. J Clin Periodontol 2015;42(Suppl 16):S71-S76

9. Van der Weijden FA, Van der Sluijs E, Ciancio SG, Slot DE. Can Chemical Mouthwash Agents Achieve Plaque/Gingivitis Control? Dent Clin North Am. 2015;59:799-829.

10. Boyle P, Koechlin A, Autier P. Mouthwash Use and the Prevention of Plaque, Gingivitis and Caries. Oral Diseases. 2014;20:1-68.

11. Claffey N. Essential oil mouthwashes: a key component in oral health management. Journal of clinical periodontology. 2003;30:22-4.

12. Lemos-Júnior CA, Villoria GEM. Reviewed evidence about the safety of the daily use of alcohol-based mouthrinses. Brazilian oral research. 2008;22:24-31.

13. Fourre T, Quieiroz D, Mordas CJ, McGuire JA Ilg D. In vitro efficacy of an alcohol-free essential oil containing mouthrinse. Journal of Dental Research 2011;Vol. 90, p. Abstr 793.

14. Ilg D, Junker L, Queiroz D, Mordas CJ, McGuire J, Ricci-Nittel D. In vivo efficacy of an alcohol-free essential oil containing mouthrinse. Journal of Dental Research 2012;Vol. 91, p. Abstr 791

15. Charles CA, Amini P, Gallob J, Shang H, McGuire JA, Costa R. Antiplaque and antigingivitis efficacy of an alcohol-free essential-oil containing mouthrinse: a 2-week clinical trial. American Journal of Dentistry. 2012;25:195-8.

16. Cortelli SC, Cortelli JR, Shang H, McGuire JA, Charles CA. Long-term management of plaque and gingivitis using an alcohol-free essential oil containing mouthrinse: a 6-month randomized clinical trial. Americal Journal of Dentistry. 2013;26:149-55.

17. American Dental Association. Seal of Acceptance Program Guidelines for Chemotherapeutic Products for Control of Gingivitis. s.l. : American Dental Association (ADA), 2011

18. Lobene RR, Weatherford T, Ross NM, Lamm RA, Menaker L. A modified gingival index for use in clinical trials. Clinical preventive dentistry. 1986;8:3-6.

19. Turesky S, Gilmore ND, Glickman I. Reduced plaque formation by the chloromethyl analogue of victamine C. Journal of Periodontology. 1970; 41:41-3.

20. Lobene RR, Soparkar P, Newman M. Use of dental floss. Effect on plaque and gingivitis. Clinical preventive dentistry. 1982;4:5-8.

21. Tanner AC, Kent R Jr, Van Dyke T, Sonis ST, Murray LA. Clinical and other risk indicators for early periodontitis in adults. Journal of periodontology. 2005; 76:573-81.

22. Ainamo J, Bay I. Problems and proposals for recording gingivitis and plaque. International dental journal. 1975;25:229-35.

23. Saxton CA, van der Ouderaa FJ. The effect of a dentifrice containing zinc citrate and Triclosan on developing gingivitis. J Periodontol Research. 1989; $24 \cdot 75-80$

24. Cortelli SC, Cortelli JR, Shang H, Costa R, Charles CA. Gingival health benefits of essential-oil and cetylpyridinium chloride mouthrinses: a 6-month randomized clinical study. Americal journal of dentistry. 2014;27:119-26.

25. Fieller EC. The distribution of the index in a normal bivariate population. Biometrika. 1932;24:428-40

26. Koch GG, Tangen CM. Nonparametric analysis of covariance and its role in noninferiority clinical trials. Drug information journal. 1999;33:1145-59.

27. Lamster IB, Alfano MC, Seiger MC, Gordon JM. The Effect of Listerine Antiseptic on Reduction of Existing Plaque and Gingivitis. Clinical preventive dentistry. 1983;5(6):12-6.

28. Van Leeuwen M, Slot D, van der Weijden $G$. The effect of an essential-oils mouthrinse as compared to a vehicle solution on plaque and gingival inflammation: a systematic review and meta-analysis. International Journal of Dental Hygiene. 2014;12:160-7.

29. Pan PC, Harper S, Ricci-Nittel D, Lux R, Shi W. In-vitro evidence for efficacy of antimicrobial mouthrinses. Journal of dentistry. 2010;38:516-20.

30. Mordas CJ, Gambogi RJ. Non-alcohol bioactive essential oil mouth rinses. 2015. US Patent 9084902:B2. https://www.google.com/patents/US9084902.

31. Mordas CJ, Queiroz DR, Tsai PB. Methods of preparing non-alcohol bioactive essential oil mouth rinses. US Patent. 2014;20140322146:A1

32. Brown WA. Expectation, the placebo effect and the response to treatment. Rhode Island medical journal. 2015;98:19-21

33. Kienle GS, Kiene $H$. The powerful placebo effect: fact or fiction? Journal of clinical epidemiology. 1997;50:1311-8.

34. Bauroth K, Charles CH, Mankodi SM, Simmons K, Zhao Q, Kumar LD. The efficacy of an essential oil antiseptic mouthrinse vs. dental floss in 
controlling interproximal gingivitis: a comparative study. The Journal of the American Dental Association. 2003;134:359-65.

35. Charles CA, Mostler K, Bartels L, Mankodi S. Comparative antiplaque and antigingivitis effectiveness of a chlorhexidine and an essential oil mouthrinse: 6-month clinical trial. Journal of clinical periodontology. 2004; 31:878-84.

36. Charles CA, McGuire JA, Qaqish J, Amini P. Increasing antiplaque/ antigingivitis efficacy of an essential oil mouthrinse over time: an in vivo study. General Dentistry. 2013;61:23-8.

37. Sharma N, Charles CH, Lynch MC, Qaqish J, McGuire JA, Galustians JG, Kumar LD. Adjunctive benefit of an essential oil-containing mouthrinse in reducing plaque and gingivitis in patients who brush and floss regularly: a six-month study. The Journal of the American Dental Association. 2004;135: 496-504.

Submit your next manuscript to BioMed Central and we will help you at every step:

- We accept pre-submission inquiries

- Our selector tool helps you to find the most relevant journal

- We provide round the clock customer support

- Convenient online submission

- Thorough peer review

- Inclusion in PubMed and all major indexing services

- Maximum visibility for your research

Submit your manuscript at www.biomedcentral.com/submit
Biomed Central 